\title{
Generation of maximally entangled states via dispersive interactions
}

\author{
Alfredo Luis* \\ Departamento de Óptica, Facultad de Ciencias Físicas, Universidad Complutense, 28040 Madrid, Spain
}

(Received 7 October 2001; published 31 January 2002)

\begin{abstract}
We propose a simple method to generate in practice the most general two-mode states with maximum entanglement. The method is based on energy-conserving dispersive interactions that can be easily implemented in different context such as quantum optical fields, quantum motion of trapped ions, or in BoseEinstein condensates.
\end{abstract}

DOI: 10.1103/PhysRevA.65.034102

PACS number(s): 03.65.Ud, 42.50.Dv

Entanglement is one of the most striking features of quantum physics. In particular it serves to demonstrate fundamental quantum properties which are far beyond the conceptual framework defined by classical physics. Leaving aside its theoretical importance we can point out promising practical applications in diverse areas such as quantum computing, teleportation, lithography, and precision measurements for example.

In this report, we are interested in states with maximum entanglement, the so-called maximally entangled states [1,2]. We show that arbitrary two-mode states having this property can be easily generated by using purely dispersive interactions (i.e., energy-conserving interactions). In comparison with other more specific proposals [2], this scheme can produce a larger class of states and is valid in very different contexts.

Throughout we will deal with a system made of two independent degrees of freedom represented by bosonic operators $a_{1}, a_{2}$ (complex amplitude or annihilation operators) obeying the standard commutation relations $\left[a_{1}, a_{1}^{\dagger}\right]$ $=\left[a_{2}, a_{2}^{\dagger}\right]=1,\left[a_{1}, a_{2}\right]=\left[a_{1}, a_{2}^{\dagger}\right]=0$. This can be describing very different physical situations such as two electromagnetic-field modes (either traveling fields or standing waves), the two-dimensional vibrational motion of a trapped ion, or two Bose-Einstein condensates. For definiteness we focus on cavity fields although this method can be translated to all the practical situations just mentioned.

The most standard examples of maximally entangled states are the two-mode states $[1,2]$

$$
\left|\eta_{n}\right\rangle=\frac{1}{\sqrt{2}}\left(|n\rangle_{1}|0\rangle_{2}+e^{i \phi_{0}}|0\rangle_{1}|n\rangle_{2}\right)
$$

where $|n\rangle_{1,2}$ denote number states in the corresponding modes, $\phi_{0}$ is a constant phase, and we assume that $n \neq 0$. These states are maximally entangled in the sense that if we find any particle in one of the modes ( $a_{1}$ or $a_{2}$ with the same probability) all the rest of the particles must be found in the same mode.

The goal is to produce the state

\footnotetext{
*Electronic address: alluis@ fis.ucm.es
}

$$
|\widetilde{\Psi}\rangle=\frac{1}{\sqrt{2}}\left(|\psi\rangle_{1}|0\rangle_{2}+e^{i \phi_{0}}|0\rangle_{1}|\psi\rangle_{2}\right),
$$

where $|\psi\rangle$ is an arbitrary state, $|0\rangle$ is the vacuum, and we assume that $\langle 0 \mid \psi\rangle=0$. This is the most general two-mode state with maximum entanglement since it is an arbitrary superposition of the states (1). It must be pointed out that this is not the most general two-mode entangled state because one of the modes is always in the vacuum. In this context we can mention that the entanglement with the vacuum may be a controversial issue concerning the nonlocal properties of single particles [3]. However, our report does not focus on this particular consequence of quantum entanglement, so it is free from such interpretational questions.

The proposed generation of the state (2) relies on the dispersive interaction between two cavity field modes of the same frequency and an effective two-level atom described by the two internal orthogonal states $|e\rangle$ and $|g\rangle$. This interaction couples $|e\rangle$ to a third level $\left|e^{\prime}\right\rangle$. If the detuning between the frequency of the cavity fields and the atomic frequency is large enough the transition probability is negligible. This nonresonant interaction produces a mutual phase shift without any energy exchange between the atom and the fields. If we assume that the strength of the atom-field coupling is the same for both modes the nonresonant interaction is described by the effective Hamiltonian

$$
H_{\mathrm{int}} \propto b_{1}^{\dagger} b_{1}|e\rangle\langle e|,
$$

where $b_{1}$ is one of the two following complex amplitude operators:

$$
b_{1}=\frac{1}{\sqrt{2}}\left(a_{1}+a_{2}\right), \quad b_{2}=\frac{1}{\sqrt{2}}\left(a_{1}-a_{2}\right),
$$

that verify the bosonic commutation relations $\left[b_{1}, b_{1}^{\dagger}\right]$ $=\left[b_{2}, b_{2}^{\dagger}\right]=1,\left[b_{1}, b_{2}\right]=\left[b_{1}, b_{2}^{\dagger}\right]=0$. This dispersive coupling can be easily implemented in practice in many different situations [4-10].

In order to generate the states (2) we consider that the whole atom-field system is initially in the state

$$
|\Psi\rangle=|\psi\rangle_{1}|0\rangle_{2}|\varphi\rangle
$$

where the atomic state $|\varphi\rangle$ is 


$$
|\varphi\rangle=\frac{1}{\sqrt{2}}(|g\rangle+|e\rangle) .
$$

After the interaction governed by the Hamiltonian (3) the joint atom-field state becomes $\left|\Psi^{\prime}\right\rangle=T|\Psi\rangle$ where

$$
T=e^{i \tau b_{1}^{\dagger} b_{1}|e\rangle\langle e|},
$$

and $\tau$ is a parameter depending on the interaction time. The interaction time can be suitably adjusted so that $\tau=\pi$ and

$$
\begin{gathered}
T|n\rangle_{1}|0\rangle_{2}|e\rangle=(-1)^{n}|0\rangle_{1}|n\rangle_{2}|e\rangle, \\
T|n\rangle_{1}|0\rangle_{2}|g\rangle=|n\rangle_{1}|0\rangle_{2}|g\rangle,
\end{gathered}
$$

and then

$$
\left|\Psi^{\prime}\right\rangle=e^{-i \pi a_{2}^{\dagger} a_{2}} \frac{1}{\sqrt{2}}\left(|\psi\rangle_{1}|0\rangle_{2}|g\rangle+|0\rangle_{1}|\psi\rangle_{2}|e\rangle\right) .
$$

After this atom-field entanglement we apply a phase-shift $\pi$ to the mode $a_{2}$ to obtain the state

$$
\left|\Psi^{\prime \prime}\right\rangle=e^{i \pi a_{2}^{\dagger} a_{2}}\left|\Psi^{\prime}\right\rangle=\frac{1}{\sqrt{2}}\left(|\psi\rangle_{1}|0\rangle_{2}|g\rangle+|0\rangle_{1}|\psi\rangle_{2}|e\rangle\right) .
$$

The target state (2) can be finally produced by quantum state reduction associated to a measurement performed on the atomic variables and described by projection on the vectors

$$
\left|\varphi_{ \pm}\right\rangle=\frac{1}{\sqrt{2}}(|g\rangle \pm|e\rangle) .
$$

From a practical perspective, this measurement can be performed by detecting the probability of the occupation of $|e\rangle$, $|g\rangle$ (by using field-ionization detectors for instance) after applying a resonant laser pulse transforming $\left|\varphi_{ \pm}\right\rangle$into $|e\rangle$, $|g\rangle$ ( $\pi / 2$ pulse). The state of the cavity field $\left|\widetilde{\Psi}_{ \pm}\right\rangle$after the measurement gives the desired state

$$
\left|\widetilde{\Psi}_{ \pm}\right\rangle=\sqrt{2}\left\langle\varphi_{ \pm} \mid \Psi^{\prime \prime}\right\rangle=\frac{1}{\sqrt{2}}\left(|\psi\rangle_{1}|0\rangle_{2} \pm|0\rangle_{1}|\psi\rangle_{2}\right) .
$$

Up to now we have been following a passive picture of the relation between modes $a$ and $b$. It is also possible to adopt an active point of view in which we deal always with the same pair of modes, say $a_{1}, a_{2}$. In such a case, the mode relation (4) corresponds to an input-output transformation actually performed on the system. Such action is represented by the unitary operator

$$
R=e^{\pi / 4\left(a_{1}^{\dagger} a_{2}-a_{2}^{\dagger} a_{1}\right)},
$$

such that

$$
R^{\dagger} a_{1} R=\frac{1}{\sqrt{2}}\left(a_{1}+a_{2}\right),
$$

$$
R^{\dagger} a_{2} R=\frac{1}{\sqrt{2}}\left(a_{2}-a_{1}\right)
$$

In this picture the target state (2) is prepared performing the above atomic measurement after applying consecutively four transformations

$$
\left|\Psi^{\prime \prime}\right\rangle=e^{i \pi a_{2}^{\dagger} a_{2}} R^{\dagger} e^{i \pi a_{1}^{\dagger} a_{1}|e\rangle\langle e|} R|\Psi\rangle .
$$

This mode coupling $R$ can be easily implemented for traveling waves by using beam splitters or phase plates. In the case of standing waves in the same cavity, $R$ can be achieved by means of phase plates controlled via electro-optical effects or via dispersive interactions with atoms crossing the cavity. For field modes in different cavities $R$ may be performed via cavity couplings such as the one proposed in Ref. [5]. Concerning the vibrational motion of trapped ions the interaction Hamiltonian (3) and the transformation (13) can be realized as proposed in Refs. [8,10] and [11], respectively. Concerning Bose-Einstein condensates it seems that the implementation of this proposal is not as feasible as for cavity fields and trapped ions. While the transformation $R$ can be realized as proposed in Ref. [12] for example, the implementation of the interaction Hamiltonian (3) may be more difficult. As a tentative and idealized scheme to reach this goal we may consider the interaction of the condensates with a quantum field mode of complex amplitude operator $a$ [13]. For large detuning the interaction becomes purely dispersive so that Eq. (3) is replaced by $H_{\text {int }} \propto b_{1}^{\dagger} b_{1} a^{\dagger} a$. In such a case the photon number states $|0\rangle,|1\rangle$ of the field mode $a$ would play the role of $|g\rangle$ and $|e\rangle$, respectively.

We illustrate the method discussed above for cavity fields considering the particular case in which $|\psi\rangle$ is a coherent state $|\psi\rangle=|\alpha\rangle$. The application of the transformation $R$ to the initial field state $|\alpha\rangle_{1}|0\rangle_{2}$ leads to

$$
R|\alpha\rangle_{1}|0\rangle_{2}=|\beta\rangle_{1}|-\beta\rangle_{2},
$$

where $| \pm \beta\rangle$ are coherent states and $\beta=\alpha / \sqrt{2}$. In the next step a two-level atom is prepared in the initial state (6). The atom crosses the cavity interacting only with the field mode $a_{1}$ during a suitable interaction time so that after leaving the cavity the joint atom-field state becomes

$$
\begin{aligned}
\left|\Psi^{\prime}\right\rangle= & e^{i \pi a_{1}^{\dagger} a_{1}|e\rangle\langle e|} R|\alpha\rangle_{1}|0\rangle_{2}|\varphi\rangle=\frac{1}{\sqrt{2}}\left(|\beta\rangle_{1}|-\beta\rangle_{2}|g\rangle\right. \\
& \left.+|-\beta\rangle_{1}|-\beta\rangle_{2}|e\rangle\right)
\end{aligned}
$$

After projection on the atomic states (11) the reduced field state is

$$
\left|\Psi_{ \pm}^{\prime}\right\rangle=\mathcal{N}_{ \pm}\left(|\beta\rangle_{1}|-\beta\rangle_{2} \pm|-\beta\rangle_{1}|-\beta\rangle_{2}\right),
$$

where $\mathcal{N}_{ \pm}$are normalization constants that can be approximated as $\mathcal{N}_{ \pm} \simeq 1 / \sqrt{2}$ if $|\alpha| \gg 1$. The final form for the desired state is achieved after performing the transformation $R^{\dagger}$ and applying a $\pi$ phase shift in mode $a_{2}$ 


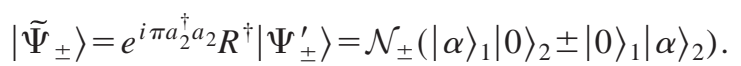

We can note that the states of the form (2) satisfy the eigenvalue equation $a_{1} a_{2}|\tilde{\Psi}\rangle=0$ so they are closely related to the so-called pair coherent states [14]. Moreover, the states (18) are examples of the so-called entangled coherent states [15]. As a matter of fact, the state (18) factorizes as the product of a Schrödinger cat state in mode $a_{1}$ and a coherent state in mode $a_{2}$. This is an example of a very general relation between Schrödinger cats and maximally entangled states [16] that implies that most methods proposed for generating Schrödinger cats can serve to generate maximally entangled states. In this sense, schemes devised to generate Schrödinger cat states and entangled coherent states based on dispersive interactions can be found in Refs. [6-8]. In particular, an all-optical dispersive generation of entangled coherent states very similar to the one discussed above has been presented in Ref. [9]. Nevertheless, we stress that the method presented here goes beyond cat states and coherent states and serves to generate arbitrary two-mode states with maximum entanglement.

Summarizing, in this report we have proposed a simple method to generate in practice arbitrary two-mode states with maximum entanglement which is valid and easily implementable in many different physical contexts.
[1] N.D. Mermin, Phys. Rev. Lett. 65, 1838 (1990).

[2] J.J. Bollinger, W.M. Itano, D.J. Wineland, and D.J. Heinzen, Phys. Rev. A 54, R4649 (1996); C.C. Gerry, ibid. 53, 2857 (1996); S.-B. Zheng and G.-C. Guo, J. Mod. Opt. 44, 963 (1997); D.J. Wineland, C. Monroe, W.M. Itano, D. Kielpinski, B.E. King, C.J. Myatt, Q.A. Turchette, and C.S. Wood, e-print quant-ph/9809028; J. Steinbach and C.C. Gerry, Phys. Rev. Lett. 81, 5528 (1998); K. Mølmer and A. Sørensen, ibid. 82, 1835 (1999); D. Jaksch, H.-J. Briegel, J.I. Cirac, C.W. Gardiner, and P. Zoller, ibid. 82, 1975 (1999); C.C. Gerry, Phys. Rev. A 61, 043811 (2000); C.A. Sackett, D. Kielpinski, B.E. King, C. Langer, V. Meyer, C.J. Myatt, M. Rowe, Q.A. Turchette, W.M. Itano, D.J. Wineland, and C. Monroe, Nature (London) 404, 256 (2000); A. Rauschenbeutel, G. Nogues, S. Osnaghi, P. Bertet, M. Brune, J.M. Raimond, and S. Haroche, Science 288, 2024 (2000); S. Raghavan, H. Pu, P. Meystre, and N.P. Bigelow, Opt. Commun. 188, 149 (2001); V. Meyer, M.A. Rowe, D. Kielpinski, C.A. Sackett, W.M. Itano, C. Monroe, and D.J. Wineland, Phys. Rev. Lett. 86, 5870 (2001); C.C. Gerry and R.A. Campos, Phys. Rev. A 64, 063814 (2001); J.A. Dunningham and K. Burnett, J. Mod. Opt. 48, 1837 (2001).

[3] D.M. Greenberger, M.A. Horne, and A. Zeilinger, in Quantum Interferometry, edited by F. De Martini, G. Deuardo, and Y. Shih (VCH, Weinheim, 1996); G. Björk, P. Jonsson, and L.L. Sánchez-Soto, Phys. Rev. A 64, 042106 (2001), and references therein.

[4] M. Brune, S. Haroche, V. Lefevre, J. M. Raimond, and N. Zagury, Phys. Rev. Lett. 65, 976 (1990); M. Brune, S. Haroche, J.M. Raimond, L. Davidovich, and N. Zagury, Phys. Rev. A 45, 5193 (1992); M. Brune, E. Hagley, J. Dreyer, X. Maitre, A. Maali, C. Wunderlich, J.M. Raimond, and S.
Haroche, Phys. Rev. Lett. 77, 4887 (1996); S. Haroche, Phys. Today 51(7), 36 (1998).

[5] J.M. Raimond, M. Brune, and S. Haroche, Phys. Rev. Lett. 79, 1964 (1997).

[6] L. Davidovich, A. Maali, M. Brune, J.M. Raimond, and S. Haroche, Phys. Rev. Lett. 71, 2360 (1993); L. Davidovich, M. Brune, J.M. Raimond, and S. Haroche, Phys. Rev. A 53, 1295 (1996).

[7] C.M. Savage, S.L. Braunstein, and D.F. Walls, Opt. Lett. 15, 628 (1990).

[8] J.C. Retamal and N. Zagury, Phys. Rev. A 55, 2387 (1997); C.C. Gerry, ibid. 55, 2478 (1997).

[9] D. Vitali, P. Tombesi, and Ph. Grangier, Appl. Phys. B: Lasers Opt. 64, 249 (1997).

[10] R.L. de Matos Filho and W. Vogel, Phys. Rev. A 58, R1661 (1998).

[11] D.J. Wineland, C. Monroe, W.M. Itano, B.E. King, D. Leibfried, C. Myatt, and C. Wood, Phys. Scr., T 76, 147 (1998).

[12] P. Bouyer and M.A. Kasevich, Phys. Rev. A 56, R1083 (1997).

[13] Z. Hepinga, L. Fuchenga, and Z. Weipingb, Phys. Lett. A 201, 397 (1995); M.G. Moore and P. Meystre, Phys. Rev. A 59, R1754 (1999); D. Jaksch, S.A. Gardiner, K. Schulze, J.I. Cirac, and P. Zoller, Phys. Rev. Lett. 86, 4733 (2001).

[14] G.S. Agarwal, Phys. Rev. Lett. 57, 827 (1986); J. Opt. Soc. Am. B 5, 1940 (1988); S.-C. Gou, J. Steinbach, and P.L. Knight, Phys. Rev. A 54, R1014 (1996); X.-M. Liu, Phys. Lett. A 279, 123 (2001).

[15] B.C. Sanders, Phys. Rev. A 45, 6811 (1992); 46, 2966 (1992); R. Filip, J. Řeháček, and M. Dušek, J. Opt. B: Quantum Semiclassical Opt. 3, 341 (2001); S.-B. Zheng, ibid. 3, 333 (2001).

[16] A. Luis, Phys. Rev. A 64, 054102 (2001). 